\title{
Nonlinear interactions between three inertial Alfvén waves
}

\author{
G. B R O D I N, L. STEN F L O and P. K. SH UK L A \\ Department of Physics, Umeå University, SE-901 87 Umeå, Sweden
}

(Received 9 May 2006)

\begin{abstract}
The resonant coupling between Alfvén waves is reconsidered. New results are found for cold magnetoplasmas where temperature effects are negligible.
\end{abstract}

The nonlinear interaction between Alfvén waves (Sagdeev and Galeev 1969; Hasegawa and Uberoi 1982; Petviashvili and Pokhotelov 1992) is a key subject within modern plasma science (Shukla 2004) that is still a matter of subtle analysis (e.g. Fedun et al. 2004; Voitenko and Goossens 2005; Shukla and Stenflo 2005). In order to put the theory on a firm theoretical basis, we shall consider the resonant interaction between three inertial Alfvén waves in a uniform cold magnetized plasma, starting our analysis from the exact expressions for the coupling coefficients in such a plasma. Considering the appropriate frequency limits, we shall then derive results that have not been presented previously.

Let us thus investigate the resonant interaction between three waves with frequencies $\omega_{j}(j=1,2,3)$ and wavevectors $\mathbf{k}_{j}$, and assume that the matching conditions

$$
\omega_{3}=\omega_{1}+\omega_{2}
$$

and

$$
\mathbf{k}_{3}=\mathbf{k}_{1}+\mathbf{k}_{2}
$$

are satisfied. The evolution of, for example, the $z$-components $\left(E_{j z}\right)$ of the wave electric field amplitudes is then governed by the three coupled bilinear equations (e.g. Stenflo 1994)

$$
\begin{aligned}
& \frac{d E_{1 z}^{*}}{d t}=\alpha_{1} E_{2 z} E_{3 z}^{*}, \\
& \frac{d E_{2 z}^{*}}{d t}=\alpha_{2} E_{1 z} E_{3 z}^{*}
\end{aligned}
$$

and

$$
\frac{d E_{3 z}}{d t}=\alpha_{3} E_{2 z} E_{1 z}
$$

where the $z$-axis is along the external magnetic field $\left(B_{0} \widehat{\mathbf{z}}\right)$, the asterisk denotes the complex conjugate, $\alpha_{j}$ are the coupling coefficients, $d / d t=\partial / \partial t+\mathbf{v}_{\mathrm{g} j} \cdot \nabla+\nu_{j}$, where $\mathbf{v}_{\mathrm{g} j}$ is the group velocity of wave $j$, and $\nu_{j}$ accounts for the linear damping rate. As shown by Stenflo and Brodin (2005), the coefficients $\alpha_{j}$ for a cold magnetoplasma 
are

and

$$
\alpha_{1,2}=\frac{M_{1,2}}{\partial D\left(\omega_{1,2}, \mathbf{k}_{1,2}\right) / \partial \omega_{1,2}} C
$$

$$
\alpha_{3}=-\frac{M_{3}}{\partial D\left(\omega_{3}, \mathbf{k}_{3}\right) / \partial \omega_{3}} C
$$

where

$$
\begin{aligned}
& C=\sum_{\sigma} \frac{q \omega_{\mathrm{p}}^{2}}{m \omega_{1} \omega_{2} \omega_{3} k_{1 z} k_{2 z} k_{3 z}} \\
& \times\left[\frac{\mathbf{k}_{1} \cdot \mathbf{K}_{1}}{\omega_{1}} \mathbf{K}_{2} \cdot \mathbf{K}_{3}^{*}+\frac{\mathbf{k}_{2} \cdot \mathbf{K}_{2}}{\omega_{2}} \mathbf{K}_{1} \cdot \mathbf{K}_{3}^{*}+\frac{\mathbf{k}_{3} \cdot \mathbf{K}_{3}^{*}}{\omega_{3}} \mathbf{K}_{1} \cdot \mathbf{K}_{2}\right. \\
& \left.-\frac{i \omega_{\mathrm{c}}}{\omega_{3}}\left(\frac{k_{2 z}}{\omega_{2}}-\frac{k_{1 z}}{\omega_{1}}\right) \mathbf{K}_{3}^{*} \cdot\left(\mathbf{K}_{1} \times \mathbf{K}_{2}\right)\right] \text {, } \\
& \mathbf{K}=-\left[\mathbf{k}_{\perp}+i \frac{\omega_{\mathrm{e}}}{\omega} \mathbf{k} \times \widehat{\mathbf{z}}\right. \\
& \left.+\left(\frac{\sum i\left(\omega_{\mathrm{c}} / \omega\right)\left[\omega_{\mathrm{p}}^{2} /\left(\omega^{2}-\omega_{\mathrm{c}}^{2}\right)\right]}{1-\left(k^{2} c^{2} / \omega^{2}\right)-\sum \omega_{\mathrm{p}}^{2} /\left(\omega^{2}-\omega_{\mathrm{c}}^{2}\right)}\right)\left(\mathbf{k} \times \widehat{\mathbf{z}}-i \frac{\omega_{\mathrm{c}}}{\omega} \mathbf{k}_{\perp}\right)\right] \\
& \times \frac{\left(1-\left(k_{\perp}^{2} c^{2} / \omega^{2}\right)-\sum \omega_{\mathrm{p}}^{2} / \omega^{2}\right) \omega^{4}}{\left(\omega^{2}-\omega_{\mathrm{c}}^{2}\right) k_{\perp}^{2} c^{2}}+k_{z} \widehat{\mathbf{z}}, \\
& D(\omega, \mathbf{k})=\left(1-\frac{k^{2} c^{2}}{\omega^{2}}-\sum \frac{\omega_{\mathrm{p}}^{2}}{\omega^{2}-\omega_{\mathrm{c}}^{2}}\right) \\
& \times\left[\left(1-\frac{k_{z}^{2} c^{2}}{\omega^{2}}-\sum \frac{\omega_{\mathrm{p}}^{2}}{\omega^{2}-\omega_{\mathrm{c}}^{2}}\right)\left(1-\frac{k_{\perp}^{2} c^{2}}{\omega^{2}}-\sum \frac{\omega_{\mathrm{p}}^{2}}{\omega^{2}}\right)-\frac{k_{\perp}^{2} k_{z}^{2} c^{4}}{\omega^{4}}\right] \\
& -\left(\sum \frac{\omega_{\mathrm{p}}^{2} \omega_{\mathrm{c}}}{\omega\left(\omega^{2}-\omega_{\mathrm{c}}^{2}\right)}\right)^{2}\left(1-\frac{k_{\perp}^{2} c^{2}}{\omega^{2}}-\sum \frac{\omega_{\mathrm{p}}^{2}}{\omega^{2}}\right),
\end{aligned}
$$

and

$$
M_{j}=\left(1-\frac{k_{j}^{2} c^{2}}{\omega_{j}^{2}}-\sum \frac{\omega_{\mathrm{p}}^{2}}{\omega_{j}^{2}-\omega_{\mathrm{c}}^{2}}\right)\left(1-\frac{k_{j z}^{2} c^{2}}{\omega_{j}^{2}}-\sum \frac{\omega_{\mathrm{p}}^{2}}{\omega_{j}^{2}-\omega_{\mathrm{c}}^{2}}\right)-\left(\sum \frac{\omega_{\mathrm{p}}^{2} \omega_{\mathrm{c}}}{\omega_{j}\left(\omega_{j}^{2}-\omega_{\mathrm{c}}^{2}\right)}\right)^{2},
$$

where $k=\left(k_{z}^{2}+k_{\perp}^{2}\right)^{1 / 2}, \mathbf{k}_{\perp}$ is the perpendicular (to $\widehat{\mathbf{z}}$ ) part of the wavevector, $\omega_{\mathrm{p}}$ is the plasma frequency ( $\omega_{\text {pe }}$ for the electrons and $\omega_{\mathrm{pi}}$ for the ions), $\omega_{\mathrm{c}}=q B_{0} / \mathrm{m}$ is the gyrofrequency, $q$ and $m$ are the particle charge and mass and $c$ is the speed of light in vacuum. For notational convenience, the subscript $\sigma$, denoting the various particle species, has been dropped out in the above formulas.

Equations (3)-(8) can be used to study the high-frequency coherent wave generation (Christiansen et al. 1981) or the energy transfer from a large-amplitude electromagnetic wave into an electrostatic electron wave and a lower-hybrid wave (e.g. Yu et al. 1974; Larsson et al. 1976; Yu et al. 1978; Murtaza et al. 1984; Shukla 
and Stenflo 1985, Stenflo 1994; Kuo 2003; Stenflo 2004). However, here we shall only adopt the above results to investigate wave couplings in the magnetohydrodynamic (MHD) regime, which includes the electron inertial effects. In a previous work (Brodin and Stenflo 1988), we noted that in the ideal MHD regime there is no coupling between three shear Alfvén waves. We will, therefore, show below that the more accurate two-fluid model gives predictions significantly different from the ideal MHD model. To demonstrate this fact, we will consider low-frequency waves where the parameters have the standard MHD ordering $\omega \sim k_{z} V_{\mathrm{A}} \ll \omega_{\mathrm{ci}}$ and $\omega_{\mathrm{ci}} \ll \omega_{\mathrm{pi}}$, where $V_{\mathrm{A}}$ is the Alfvén speed. However, in contrast to the ideal MHD, here we shall also allow for large perpendicular wavenumbers, reaching up to $k_{\perp} \sim \omega_{\text {pe }} / c \equiv \lambda_{\mathrm{e}}^{-1}$, or even $k_{\perp} \gg \lambda_{\mathrm{e}}^{-1}$, where $\lambda_{\mathrm{e}}$ is the electron skin depth. Applying this scaling to (7), we find that the usual shear Alfvén waves are modified to inertial Alfvén waves, with frequencies

$$
\omega \simeq \frac{k_{z} V_{\mathrm{A}}}{\left(1+k_{\perp}^{2} \lambda_{\mathrm{e}}^{2}\right)^{1 / 2}} .
$$

Next, we assume that all the three interacting inertial Alfvén waves are described by (9). In order to keep contact with the ideal MHD regime where $\omega \simeq k_{z} V_{\mathrm{A}}$, we will allow for $k_{\perp}^{2} \lambda_{\mathrm{e}}^{2} \ll 1$ as well as for $k_{\perp}^{2} \lambda_{\mathrm{e}}^{2} \sim 1$. Applying the above assumptions to (6), we thus introduce the approximations

$$
\mathbf{K}_{\mathrm{e}} \simeq-\frac{i \omega}{\omega_{\mathrm{ce}}} \frac{\left(1+k_{\perp}^{2} \lambda_{\mathrm{e}}^{2}\right)}{k_{\perp}^{2} \lambda_{\mathrm{e}}^{2}} \mathbf{k} \times \widehat{\mathbf{z}}+k_{z} \widehat{\mathbf{z}}
$$

and

$$
\mathbf{K}_{\mathrm{i}} \simeq-\frac{i \omega}{\omega_{\mathrm{ci}}} \frac{\left(1+k_{\perp}^{2} \lambda_{\mathrm{e}}^{2}\right)}{k_{\perp}^{2} \lambda_{\mathrm{e}}^{2}} \mathbf{k} \times \widehat{\mathbf{z}}+k_{z} \widehat{\mathbf{z}} .
$$

Substituting (10) and (11) into (5), we find that the ion contribution is negligible compared to the electron contribution, and that the coupling coefficient reduces to

$$
C_{\text {IAW }}=\frac{q_{\mathrm{e}} \omega_{\mathrm{pe}}^{2}}{m_{\mathrm{e}} \omega_{1} \omega_{2} \omega_{3}}\left(\frac{k_{1 z}}{\omega_{1}}+\frac{k_{2 z}}{\omega_{2}}+\frac{k_{3 z}}{\omega_{3}}\right)
$$

and that the dispersion relation (7) can be approximated by

$$
D(\omega, \mathbf{k})=-\frac{c^{4}\left(\omega^{2}-k^{2} V_{\mathrm{A}}^{2}\right)\left(k_{\perp}^{2} c^{2}+\omega_{\mathrm{pe}}^{2}\right)}{\omega^{6} V_{\mathrm{A}}^{4}}\left[\omega^{2}-\frac{k_{z}^{2} V_{\mathrm{A}}^{2}}{1+k_{\perp}^{2} c^{2} / \omega_{\mathrm{pe}}^{2}}\right] .
$$

Similarly (8) reduces to

$$
M=\frac{c^{4}}{\omega^{4} V_{\mathrm{A}}^{4}}\left(\omega^{2}-k^{2} V_{\mathrm{A}}^{2}\right)\left(\omega^{2}-k_{z}^{2} V_{\mathrm{A}}^{2}\right) .
$$

Thus, we have

$$
\alpha_{1,2}=\frac{\omega_{1,2}^{3} k_{1,2 \perp}^{2} c^{2}}{2\left(k_{1,2 \perp}^{2} c^{2}+\omega_{\mathrm{pe}}^{2}\right) \omega_{\mathrm{pe}}^{2}} C_{\mathrm{IAW}}
$$

and

$$
\alpha_{3}=-\frac{\omega_{3}^{3} k_{3 \perp}^{2} c^{2}}{2\left(k_{3 \perp}^{2} c^{2}+\omega_{\mathrm{pe}}^{2}\right) \omega_{\mathrm{pe}}^{2}} C_{\mathrm{IAW}} .
$$

For an easy comparison with the MHD result, it is convenient to eliminate $E_{z}$ and to work with the wave magnetic field amplitudes. The magnitude of the magnetic 
field perturbation is thus

$$
B_{j}=\frac{E_{j z}}{\omega k_{j \perp} \lambda_{\mathrm{e}}^{2}},
$$

where, to a good approximation, the wave magnetic field is directed in the $-\mathbf{k} \times \widehat{\mathbf{z}}-$ direction. The coupled mode equations $(3 \mathrm{a}-\mathrm{c})$ can, therefore, be rewritten as

$$
\frac{d B_{1,2}}{d t}=\frac{\omega_{1,2} k_{1 \perp} k_{2 \perp} k_{3 \perp} V_{\mathrm{A}}^{2}}{2\left(k_{1,2 \perp}^{2}+\lambda_{\mathrm{e}}^{-2}\right) \omega_{\mathrm{ci}}}\left(\frac{k_{1 z}}{\omega_{1}}+\frac{k_{2 z}}{\omega_{2}}+\frac{k_{3 z}}{\omega_{3}}\right) \frac{B_{2,1}^{*} B_{3}}{B_{0}}
$$

and

$$
\frac{d B_{3}}{d t}=-\frac{\omega_{3} k_{1 \perp} k_{2 \perp} k_{3 \perp} V_{\mathrm{A}}^{2}}{2\left(k_{3 \perp}^{2}+\lambda_{\mathrm{e}}^{-2}\right) \omega_{\mathrm{ci}}}\left(\frac{k_{1 z}}{\omega_{1}}+\frac{k_{2 z}}{\omega_{2}}+\frac{k_{3 z}}{\omega_{3}}\right) \frac{B_{1} B_{2}}{B_{0}} .
$$

Choosing wave 3 as a pump wave, the maximum growth rate $\gamma_{\max }$ deduced from (18) is thus of the order of

$$
\gamma_{\max } \sim \frac{\omega_{3} k_{3 \perp} V_{\mathrm{A}}}{\omega_{\mathrm{ci}}} \frac{\left|B_{3}\right|}{B_{0}},
$$

where the fastest growth occurs for decay products that are inertial Alfvén waves with wavenumbers $k_{1,2 \perp}$ of the order of $\lambda_{\mathrm{e}}^{-1}$. We note that for decay into standard ideal Alfvén waves with $k_{1,2 \perp} \sim k_{1,2 z}=\omega_{1,2} / V_{\mathrm{A}}$, the growth rate thus is reduced by a factor $\sim \omega_{3}^{2} m_{\mathrm{e}} / \omega_{\mathrm{ci}}^{2} m_{\mathrm{i}}$. As a special limit of (20), we consider a pump wave that is a standard ideal Alfvén wave with $k_{3 \perp} \sim k_{3 z}=\omega_{3} / V_{\mathrm{A}}$. This gives

$$
\gamma_{\max } \sim \frac{\omega_{3}^{2}}{\omega_{\mathrm{ci}}} \frac{\left|B_{3}\right|}{B_{0}}
$$

As another example, we let the pump wave be an inertial Alfven wave with perpendicular wavenumber $k_{3 \perp} \sim \lambda_{\mathrm{e}}^{-1}$, in which case (20) reduces to

$$
\gamma_{\max } \sim \omega_{3} \frac{\left|B_{3}\right|}{B_{0}}\left(\frac{m_{\mathrm{i}}}{m_{\mathrm{e}}}\right)^{1 / 2}
$$

To summarize, we have considered nonlinear interactions of inertial Alfvén waves using results from the exact two-fluid equations for a cold magnetoplasma. We note that for an Alfvén pump wave in the inertial regime $\left(k_{3 \perp} \sim \lambda_{\mathrm{e}}^{-1}\right)$ the maximum growth rate for decay into inertial Alfvén waves is larger than the usual MHD growth rates (including interaction between all kinds of the ideal MHD waves) by a factor of the order of $\left(m_{\mathrm{i}} / m_{\mathrm{e}}\right)^{1 / 2}$. Furthermore, for an ordinary Alfvén pump wave $\left(k_{3 \perp} \ll \lambda_{\mathrm{e}}^{-1}\right)$, we point out that the cold ideal MHD theory does not allow for resonant decay processes at all (Brodin and Stenflo 1988), since the Manley-Rowe relations prevent decay into modes of higher frequencies and the coupling coefficients for interaction with two other Alfvén waves are zero in the framework of the ideal MHD model. Thus, the growth rate found in (21) is the fastest decay possible in a cold magnetoplasma for an Alfvénic pump in the ideal MHD regime $\left(k_{3 \perp} \ll \lambda_{\mathrm{e}}^{-1}\right)$. Furthermore, since the decay products have short scalelengths, we note that the ideal MHD equations are unable to describe the nonlinear evolution, even if the initial conditions lie well inside the usual validity conditions of those equations. We point out that resistivity eventually leads to dissipation of the shorter scale waves. Thus, we conclude that the parametric processes considered here can be important for understanding the heating of low-beta plasmas such as those in the Earth's auroral zone. Finally, we stress that high-beta plasmas require a separate 
analysis (Brodin and Stenflo 1990) and that extensions to three-wave interactions in a turbulent plasma (Vladimirov and Yu 2004) are comparatively straightforward.

\section{References}

[1] Brodin, G. and Stenflo, L. 1988 J. Plasma Phys. 39, 277.

[2] Brodin, G. and Stenflo, L. 1990 Contr. Plasma Phys. 30, 413.

[3] Christiansen, P. J., Jain, V. K. and Stenflo, L. 1981 Phys. Rev. Lett. 46, 1333.

[4] Fedun, V. N., Yukhimuk, A. K. and Voitsekhovskaya, A. D. 2004 J. Plasma Phys. 70, 699 .

[5] Hasegawa, A. and Uberoi, C. 1982 The Alfvén Wave (DOE Review Series-Advances in Fusion Science and Engineering). Washington D.C.: U.S. Department of Energy.

[6] Kuo, S. P. 2003 Phys. Lett. A 307, 244.

[7] Larsson, J., Stenflo, L. and Tegeback, R. 1976 J. Plasma Phys. 16, 37.

[8] Murtaza, G. and Shukla, P. K. 1984 J. Plasma Phys. 31, 423.

[9] Petviashvili, V. I. and Pokhotelov, O. A. 1992 Solitary Waves in Plasmas and in the Atmosphere. Philadelphia, PA: Gordon and Breach.

[10] Sagdeev, R. Z. and Galeev, A. A. 1969 Nonlinear Plasma Theory. New York: Benjamin.

[11] Shukla, P. K. and Stenflo, L. 1985 Phys. Fluids 28, 1576.

[12] Shukla, P. K. (ed.) 2004 Nonlinear physics in action. Phys. Scripta T113, 1.

[13] Shukla, P. K. and Stenflo, L. 2005 Phys. Plasmas 12, 084502.

[14] Stenflo, L. 1994 Phys. Scripta T50, 15.

[15] Stenflo, L. 2004 Phys. Scripta T107, 262.

[16] Stenflo, L. and Brodin, G. 2005 J. Plasma Phys. 72, 143.

[17] Vladimirov, S. V. and Yu, M. Y. 2004 Phys. Scripta T113, 32.

[18] Voitenko, Y. M. and Goossens, M. 2005 Phys. Rev. Lett. 94, 135003.

[19] Yu, M. Y., Spatschek, K. H. and Shukla, P. K. 1974 Z. Naturf. A 29, 1736.

[20] Yu, M. Y., Shukla, P. K. and Spatschek, K. H. 1978 J. Plasma Phys. 20, 182. 